\title{
Quasi-phase matching of harmonics generating in laser plasmas: experiment and theory
}

\author{
Sergey Stremoukhov ${ }^{1,2, *}$, Rashid Ganeev ${ }^{3,4}$, and Anatoli Andreev ${ }^{1}$ \\ ${ }^{1}$ Faculty of Physics, Lomonosov Moscow State University, 119991 Moscow, Russia \\ ${ }^{2}$ National Research Centre "Kurchatov Institute", 123182 Moscow, Russia \\ ${ }^{3}$ Physics Department, American University of Shariah, 26666 Shariah, UAE \\ ${ }^{4}$ Faculty of Physics, Voronezh State University, 394006 Voronezh, Russia
}

\begin{abstract}
We compare the results of experimental and theoretical studies of the quasi-phase matching (QPM) of high-order harmonic generation (HHG) during propagation of two-color femtosecond pulses through the indium multi-jet plasmas. The role of number of coherent zones and size of plasma jets on the HHG efficiency at QPM conditions is analysed. We demonstrate the enhancement of the HHG efficiency due to QPM during laser-plasma interaction.
\end{abstract}

Different regimes of laser-plasma interaction attract interest due to possibility of conversion of longer-wavelength laser emission to extreme ultraviolet (XUV) region. The various methods have been used to enhance the efficiency of this process (please, see introduction part of the [1]). One of the approach used in [2], though attractive in the case of ablation of extended targets, has a significant drawback related with the phase mismatch between interacting waves of driving pulse and harmonic emission when the length of medium exceeds so-called coherence length for the generated harmonic order. To diminish role of phase mismatch in laser-produced plasmas (LPP) on HHG efficiency the QPM concept has been introduced. In this case the growing difference between the phases of two waves is nullified by using the modulated shape of LPP [3]. The method is based on the division of extended plasma onto the set of separated plasma jets by inserting the multi-slit mask (MSM) in front of ablating surface [4], or by using the perforated targets [5].

In the study of QPM conditions it is usually assumed that the target is in some fixed state with some given degree and level of ionization. This approximation is more or less suitable to describe the propagation of the driving laser wave, however it is not suitable to describe the interaction of harmonics with plasmas, since harmonics are emitted in different spatial points of nonlinear optical medium during the whole action of driving laser field. The model that takes into account the propagation of the driving laser field and at the same time includes the quantum mechanical description of atomic electron evolution at QPM conditions has recently been proposed in Ref. [6]. Recently, the model was applied to study the QPM conditions in perforated silver plasma [7]. In this paper, we extend the latter research by focusing in comparing conditions of QPM in indium multi-jet plasmas.

The $70 \mathrm{fs}$ near infrared pulses $(1320 \mathrm{~nm}+660 \mathrm{~nm})$ from optical parametric amplifier for harmonic generation are used. The 340 ps pulses were focused using $300-\mathrm{mm}$ focal length

\footnotetext{
*Corresponding author: sustrem@gmail.com
} 
cylindrical lens on the surface of ablating target placed in the vacuum chamber to form the homogeneous extended plasma $(5 \mathrm{~mm})$. To produce multi-jet plasmas (MJP) the MSM was installed between cylindrical lens and target. The width of each slit was varied between 0.3 and $0.8 \mathrm{~mm}$ with similar distance between slits. The details of experimental setup and the parameters of the experimental conditions are presented in Refs. [7].

For numerical investigations we used the 1D model described in Ref. [6]. Within this model the nonlinear medium is simulated by $1 \mathrm{D}$ chain of atoms oriented along the propagation direction of laser pulses. The response of each atom in the chain is calculated using the non-perturbative theory [8]. Generated harmonic radiation from each atom is calculated in the far far-field zone by a summation over chain of atoms $[6,9]$. We have studied the dependence of harmonic spectra on the individual jet size under conditions of the constant length $(\sim 5 \mathrm{~mm})$ of indium plasma. The number of jets changed with variation of their length.
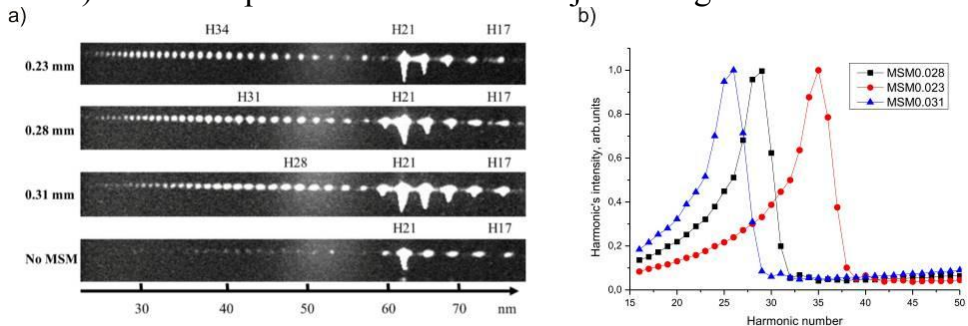

Fig. 1. Raw images of harmonic spectra from indium MJP with the single plasma jet sizes of (top to bottom) $0.23,0.28,0.31 \mathrm{~mm}$ pumped by two-color pulses: a) experiment [7], b) calculation.

Three strongly modulated and enhanced spectra of the groups of odd and even harmonics of two-color laser field compared to the case of extended indium plasma are shown in Fig. 1(a). Three upper spectra containing significantly enhanced groups of harmonics centered at $\mathrm{H} 34$, $\mathrm{H} 31$, and $\mathrm{H} 28$ were observed in the 11-, 9-, and 8-jet indium plasmas, with the sizes of single plasmas of $0.23,0.28$, and $0.31 \mathrm{~mm}$ respectively. Fig. 1 (b) demonstrates the corresponding numerically obtained normalized spectra. The positions of the peaks in the calculated spectra are nicely coinciding with the ones from the experiment.

Meanwhile, the bottom spectrum shows the HHG from the homogeneous extended indium plasma produced at similar conditions. The enhancement factors of strongly modulated harmonics with regard to those generated in the case of homogeneous plasma were measured to be 38, 36, and 27 respectively. Spectra of Fig. 1(a) show strong resonanceinduced enhancement of $\mathrm{H} 21$ and $\mathrm{H} 20$ [10].

In this paper, we extended our latter investigations [7] to the study of the QPM in multijet indium plasma. We have experimentally and numerically demonstrated the enhancement of the group harmonics yield for which conditions of QPM became most favorable.

The theoretical part of the work was partially supported by the RFBR (pr. № 18-02-40014).

\section{References}

1. S. Stremoukhov, A. Andreev, B. Vodungbo, et al. Phys. Rev. A, 94, 013855 (2016).

2. R. A. Ganeev, M. Suzuki, H. Kuroda, Phys. Plasmas 21, 053503 (2014).

3. R. A. Ganeev, M. Suzuki, H. Kuroda, Phys. Rev. A 89, 033821 (2014).

4. R. A. Ganeev, J. Appl. Phys. 119, 113104 (2016).

5. R. A. Ganeev, M. Suzuki, S. Yoneya, H. Kuroda, Laser Phys. 24, 115405 (2014).

6. S. Yu. Stremoukhov, A. V. Andreev, Laser Phys. 28, 035403 (2018).

7. R.A. Ganeev, S.Y. Stremoukhov, A.V. Andreev, et al., Appl. Sci., 9, 1701 (2019)

8. A. V. Andreev, S. Yu. Stremoukhov, O. A. Shoutova, Eur. Phys. J. D 66, 16 (2012).

9. S. Yu. Stremoukhov, A. V. Andreev, Laser Phys. Lett. 12, 015402 (2015).

10. R. A. Ganeev, Z. Wang, P. Lan, et al., Phys. Rev. A 93, 043848 (2016). 